\title{
Identidad y alteridad en libros de texto escolares: un estudio de caso sobre la representación de fl-findalus
}

\author{
Identity and Otherness in School Textbooks: A Case Study on the Representation \\ of Al-findalus \\ Identidade e alteridade em liuros de texto escolares: um estudo de caso sobre \\ a representação de fl-Andalus
}

\author{
Irina Rasskin-Gutman* \\ Universidad de Extremadura \\ Ignacio Brescó de Luna** \\ Aalborg University
}

Doi: http://dx.doi.org/10.12804/revistas.urosario.edu.co/apl/a.7942

\section{Resumen}

El nacionalismo, y la construcción social de la identidad nacional, implica una ideología de la primera persona del plural, donde no puede haber un nosotros sin un ellos. En este artículo se analizan las formas de producción de alteridad - entendida como la otra cara de la identidad - articuladas en la representación de Al-Andalus en un libro de texto de Ciencias Sociales, utilizado en un centro de enseñanza secundaria de la Comunidad de Madrid, España. Partiendo del concepto de 'schematic narrative templates', el objetivo es examinar en qué medida la enseñanza de Al-Andalus sigue promoviendo una narrativa con fuerte arraigo en la memoria colectiva y el modo de entender la identidad española en unas aulas con estudiantes procedentes de diversos contextos nacionales y religiosos. Los resultados muestran cómo el término "Reconquista" sigue apareciendo, en ocasiones, como un hito en el relato, proyectando una cierta continuidad histórica entre los grupos de pobladores cristianos que lucharon contra los musulmanes y los españoles actuales. Por su parte, el pasado musulmán de la Península Ibérica sigue representándose en clave de alteridad, ya sea en términos negativos como "otro-exótico-amenazante" o positivos como "otro-exótico-admirable". El artículo concluye con algunas reflexiones sobre la enseñanza de la historia en aulas cada vez más multiculturales.

Palabras clave: identidad, alteridad, Al-Andalus, schematic narrative template, análisis del discurso, psicología cultural.

\section{fibstract}

Nationalism, and the social construction of national identity, involves an ideology of the first person plural, where there can be no us without a them. The present paper

* Universidad de Extremadura, España.

** Dirigir correspondencia a Ignacio Brescó de Luna, Aalborg University, Dinamarca. Correo electrónico: ignacio@hum.aau.dk

Para citar este artículo: Rasskin-Gutman, I, \& Brescó de Luna, I (2019). Identidad y alteridad en libros de texto escolares: un estudio de caso sobre la representación de Al-Andalus. Avances en Psicología Latinoamericana, 37(3), 471-488. Doi: http://dx.doi. org/10.12804/revistas.urosario.edu.co/apl/a.7942 
features a study where we analyze how different forms of otherness -understood as the other side of identity- are articulated through the representation of Al-Andalus in a textbook of Social Sciences used in a secondary school in the Community of Madrid, Spain. Drawing on the concept of schematic narrative templates, we aim to examine the extent to which the teaching of Al-Andalus, in classrooms with students from different national and religious backgrounds, keeps on promoting a narrative deeply rooted in Spain's collective memory and national identity. The results show that the term "Reconquista" continues to appear, on occasions, as a milestone in the storyline, thus projecting a certain historical continuity between the groups of Christian settlers who fought against the Muslims and the Spaniards today. As for the Muslim past of the Iberian Peninsula, it tends to be framed in terms of alterity, whether in a negative light as the "exotic-threatening-other" or in more positive tone, as the "exotic-admirable-other. The article concludes with some reflections on the teaching of history in increasingly multicultural classrooms.

Keywords: Identity, otherness, Al-Andalus, schematic narrative template, discursive analysis, cultural psychology.

\section{Resumo}

O nacionalismo e a construção social da identidade nacional implicam uma ideologia da primeira pessoa do plural, onde não pode haver um nós sem um eles. Neste artigo se analisam as formas de produção de alteridade, entendida como a outra cara da identidade, articuladas na representação de Al-Andalus em um livro de texto de Ciências Sociais utilizado em um centro de ensino secundário da Comunidade de Madrid, Espanha. Partindo do conceito de schematic narrative templates, o objetivo é examinar em que medida o ensino de Al-Andalus continua promovendo uma narrativa com forte arraigo na memória coletiva e o modo de entender a identidade espanhola em umas aulas com estudantes procedentes de diversos contextos nacionais e religiosos. Os resultados mostram como o termo "Reconquista" continua a aparecer, em ocasiões, como um marco no relato, projetando uma certa continuidade histórica entre os grupos de povoadores cristãos que lutaram contra os muçulmanos e os espanhóis atuais. À sua vez, o passado muçulmano da Península Ibérica continua representando-se em chave de alteridade, seja em termos negativos "outro-exótico-ameaçante" seja em positivos como "outro-exótico-admirável”. O artigo conclui com algumas reflexões sobre o ensino da história em aulas cada vez mais multiculturais.

Palavras-chave: identidade, alteridade, Al-Andalus, schematic narrative template, análise do discurso, psicologia cultural.

The problem Spain has with Al Qaeda and Islamic terrorism did not begin with the Iraq Crisis. In fact, it has nothing to do with government decisions. You must go back no less than 1,300 years, to the early 8 th century, when Spain recently invaded by the Moors refused to become just another piece in the Islamic world and began a long battle to recover its identity. This Reconquest process was very long, lasting some 800 years. However, it ended successfully. There are many radical Muslims who continue to recall that defeat, many more than any rational Western mind might suspect. Osama Bin Laden is one of them. His first statement after $11^{\text {th }}$ September -I repeat, the $11^{\text {th }}$ September- did not begin by referring to New York or Iraq. His first words were to lament the loss of Al-Andalus - Moorish Medieval Spain - and compare it to the occupation of Jerusalem by the Israelis. (Aznar, 2004)

Esta cita del expresidente del gobierno español José María Aznar —extraída de su discurso pronunciado en la Universidad de Georgetown, 18 meses después del comienzo de la segunda Guerra de Irak y pasados 6 meses de los atentados de Madrid de $2004^{1}$ — pone encima de la mesa algunas cuestiones

Atribuidos a Al-Qaeda, constituyen hasta la fecha el mayor atentado yihadista en suelo europeo con casi 200 víctimas mortales. Conviene señalar que, inicialmente, el gobierno 
acerca de la (re)construcción, uso y consumo de eventos históricos con fines identitarios que van a tratarse en este trabajo. Destaca, en primer lugar, el elemento identitario, reflejado en el protagonismo de un supuesto actor colectivo (España) enfrentado a diversos representantes de un mundo considerado como ajeno: el mundo islámico. Aznar habla del pasado, contando la historia épica de cómo, hace muchos años, "España", tras ocho siglos de lucha, logró reconquistar su identidad arrebatada por "los moros". Pero también habla del presente, en el que se ubica su discurso, recordando que el problema al que se enfrentaba España en el año 2004 , tras los atentados del $11 \mathrm{M}$, lejos de responder a determinadas decisiones tomadas por su gobierno, se enmarcaría en la vieja batalla de la identidad que todavía se estaría librando. Con ello, el expresidente del gobierno español estaría recordando la temática principal de una trama que retrotrae siglos atrás en la historia; una trama abierta e irresuelta, pero al mismo tiempo fácil de entender y seguir por una gran mayoría de sus conciudadanos, al enfrentar a los mismos actores colectivos, definidos en términos esencialistas: "nosotros, los españoles-cristianos" contra "ellos, los árabes-musulmanes". Así, mediante este mismo discurso, se estaría proporcionando una suerte de guion, no solo para interpretar el escenario presente, sino también, para orientar al pueblo en él de cara al futuro. Un guion que, como tal, indicaría qué papel deben representar como españoles y qué papel cabría esperar de los antagonistas históricos, es decir, de los representantes del mundo islámico.

A este tipo de narrativas subyacen unos planteamientos identitarios muy particulares, en los que la "españolidad" queda representada por un

Aznar se negó a atribuir la responsabilidad de los atentados al integrismo islámico para atribuírsela al grupo terrorista ETA, transmitiendo con ello dos formas de alteridad en función del contexto: el independentismo vasco en el ámbito nacional y el integrismo islámico en el contexto internacional, ante una audiencia probablemente familiarizada con la idea de choque de civilizaciones de Samuel Huntington (1996). imaginario carente de diversidad a partir del silenciamiento u ocultamiento de aquellos aspectos de la realidad que incomodan o que no coinciden con el imaginario previsto. Se defiende, así, una idea unívoca, esencialista y atemporal de la identidad, en este caso española, en lugar de otra de carácter dinámica, híbrida y socio-histórica y culturalmente situada (Hall \& du Gay, 1996). Dentro del panorama político español actual, especialmente durante las elecciones generales de 2019, han irrumpido partidos de ultra derecha, en parte como reacción a la creciente diversidad religiosa y lingüístico-cultural en el territorio nacional. Dichos partidos retoman, precisamente, la retórica de la "Reconquista" para reivindicar una vuelta a una supuesta "pureza identitaria" (Moreno, 2019), empleando para ello formas simplificadas de dar sentido al pasado, presente y futuro, en la línea del discurso de Aznar.

El presente trabajo toma la enseñanza escolar del periodo histórico de Al-Andalus como pretexto para la reflexión sobre la construcción identitaria. Bajo esta etiqueta histórica, se alude al periodo musulmán en la Península Ibérica desde el año 711 hasta 1942, fecha de la toma del reino Nazarí de Granada por los Reyes Católicos; un periodo tradicionalmente enseñado en las escuelas bajo el nombre de Reconquista, por medio del cual, tiende a interpretarse el mundo musulmán como "el otro" y su presencia en la península como un largo paréntesis de ocho siglos. En este estudio, se analizan las formas de producción de alteridad — entendida como la otra cara de la identidad - articuladas mediante la representación de Al-Andalus/Reconquista en un libro de texto de Ciencias Sociales, utilizado en un centro de enseñanza secundaria de la Comunidad de Madrid. El objetivo es examinar en qué medida la enseñanza de Al-Andalus sigue promoviendo una narrativa con fuerte arraigo en la memoria colectiva y el modo de entender la identidad española en unas aulas multiculturales con estudiantes procedentes de diversos contextos nacionales y religiosos. 
Estas cuestiones son abordadas desde la llamada psicología cultural, una rama de la Psicología centrada en el papel mediador de los artefactos y prácticas culturales sobre distintos fenómenos psicológicos (Cole, 1996/1999; Rosa \& Valsiner, 2018; Vygotsky, 1978). Desde esta perspectiva teórica, se abordará el papel de la enseñanza de la historia en las escuelas, en tanto actividad institucionalmente reglada, generadora de determinadas prácticas de producción de identidad (y alteridad), mediadas por ciertas formas oficiales de narrar y tematizar el pasado. Asimismo, para el análisis de los contenidos del libro de texto, se emplean conceptos procedentes del análisis del discurso (van Dijk, 2003; Martín Rojo, 2008) y del análisis visual (van Leeuwen, 2001). Se concluye señalando la necesidad de tomar consciencia sobre el papel mediador de las narrativas sobre el pasado, a fin de evitar naturalizar determinadas esencias identitarias en un futuro cada vez más complejo y global.

\section{Memoria, identidad y alteridad desde la psicología cultural}

La psicología cultural tiene como principal foco el efecto mediador, derivado de la incorporación y uso de distintos artefactos culturales sobre las formas de interpretar el mundo y de actuar en él. Se parte de la premisa, expuesta por Wertsch (2002), según la cual "el mismo hecho de ser humano implica el uso de herramientas culturales o instrumentos mediacionales suministrados por un determinado entorno socio-cultural" (p. 11). Como señala Vygotsky (1978), el uso y continua renovación de tales artefactos ha permitido a los humanos rebasar los límites del desarrollo puramente biológico y expandir así sus funciones psicológicas, desarrollando, en consecuencia, nuevas formas de comportamiento. Por ejemplo, la expansión en la capacidad de memoria, tanto en la historia de la humanidad como en la ontogenia, puede explicarse por la incorporación de diversas tecnologías y artefactos culturales, como la construcción de memoriales (Brescó \& Wagoner, 2019), la transmisión de narrativas o la simple fragmentación de información en unidades manejables.

El interés acerca del papel mediador de las narrativas ha ido ganando protagonismo en Psicología (Bruner, 1986), especialmente en el estudio sobre la relación entre la dimensión narrativa de la memoria autobiográfica y la identidad, en línea con lo que Ricoeur (1991) denomina 'identidad narrativa'. Igualmente, ha cobrado relevancia el enfoque narrativo en el estudio de la identidad y la memoria colectiva, esto es, la relación afectiva que una comunidad tiene con su pasado, independientemente de cuán distante o incluso mítico dicho pasado pueda ser (Halbwachs, 1950/1980). El enfoque de la psicología cultural en este último campo ha pivotado en gran medida sobre el concepto de 'schematic narrative template' (Wertsch, 2002), formas simplificadas de dar sentido al pasado, presente y futuro de una determinada comunidad en torno a un mismo tema narrativo que se utiliza y reactualiza recurrentemente en distintos momentos históricos, como por ejemplo la temática centrada en la lucha por la libertad en los EE.UU. (Wertsch \& O’Connor, 1994).

Carretero y Bermúdez (2012) destacan seis aspectos principales de las narrative templates: (1) la presencia de un sujeto histórico homogéneo y permanente en el tiempo; (2) una conceptualización esencialista de la identidad, la nación, y el territorio nacional; (3) la presencia de una lógica binaria, articulada en el enfrentamiento entre "nosotros" y "ellos"; (4) la atribución de juicios morales positivos y negativos; (5) la presencia de figuras históricas heroicas; (6) una visión monocausal o teleológica de los acontecimientos históricos. En resumen, el papel de estas formas narrativas, en tanto discursos dominantes en una determinada comunidad, resulta fundamental en el mantenimiento de ciertos estilos de pensamiento histórico, actuando, a su vez, como guiones 
desde los que se puedan imaginar tanto la identidad colectiva, como la alteridad (van Alphen $\&$ Carretero, 2015).

Un elemento central en la transmisión institucional de este tipo de narrativas han sido los libros de texto escolares, relatos históricos eminentemente distintos a los de la historia como disciplina académica, al ser el resultado de una 'transposición didáctica' (Chevallard, 1991) que implica, en ocasiones, una deformación profunda, incluso contraria al ideal historiográfico. Como señala Carretero (2007), contrariamente a su supuesto papel en el fomento del pensamiento crítico, los contenidos de los libros de texto tienden a transmitir una "narración oficial de la experiencia del pasado común, a los que se agrega una importante carga emotiva destinada a crear identificación" (p. 39). ${ }^{2}$ El consumo de tales narraciones fomentaría, en este sentido, una interpretación nacional de la historia (Brescó, 2008), entendiéndose la nación como protagonista e hilo conductor entre el pasado y el presente. Un protagonista al que se le atribuyen intenciones y hazañas, así como supuestos enemigos históricos, asumidos como propios en primera persona del plural. Y es que, como señala Billig (1995), la primera persona del plural necesita de un ellos para contraponer al nosotros, si bien no siempre como algo necesariamente hostil o antagónico (Barth, 1969/1976). Desde una perspectiva dialógica (Bakhtin, 1981), podría decirse que toda comunidad imaginada (Anderson, 1983) requiere de una alteridad imaginada, siendo esta última un elemento constitutivo de la primera.

En el caso español, el proceso de nacionalización de las masas (Mosse, 1975) por medio de la enseñanza de la historia no empezó de forma sistemática hasta los años veinte del siglo pasado, durante la dictadura de Primo de Ribera (Quiroga,

Encontramos, sin embargo, otros autores (Wagner, Kello, y Saki, 2018) que sostienen que este no sería siempre el caso en algunos países.
2007). En ausencia de un imperio colonial moderno, el relato nacional se construyó sobre el pasado imperial de España y su legado católico. En este sentido, 1492 fue considerado un año clave en la historia nacional, por ser la fecha del llamado "descubrimiento" de América y la culminación de la llamada "Reconquista". Tras la Guerra Civil Española (1936-1939), la dictadura de Franco recurrió al nacional-catolicismo de Primo de Ribera como el principio definitorio de la nacionalidad española moderna. En consonancia, los libros de texto de la posguerra se centraron en "las características de la misión histórica de España [y] su servicio a los altos valores de la concepción católica de la vida" (Boyd, 1997, p. 288; véase también Álvarez Junco, 2002). Un claro ejemplo de ello, se encuentra en el siguiente fragmento introductorio al programa de Historia de España de la Enciclopedia Álvarez, manual utilizado durante la dictadura franquista, fundamentalmente entre los años 1954 y 1966:

España es una de las naciones que más ha contribuido a la civilización del mundo y que mayor influencia ha tenido en la historia universal. Para probarlo, basta citar cuatro hechos: la defensa que en la Reconquista hizo de Europa, interponiendo generosamente su pecho entre ella y la cimitarra de los árabes; el descubrimiento, colonización y civilización de América; el heroísmo desplegado en la Guerra de Independencia que contribuyó a la salvación de Europa del cesarismo de Napoleón, y el no menos heroico sacrificio llevado a cabo en el Alzamiento Nacional, ya que gracia a él el comunismo no impera hoy en buena parte del mundo [...] Esta España es tu Patria. Conoce su historia. Toma de ella los ejemplos virtuosos y heroicos que tus antepasados te brindan a cada paso y procura ser en todas las ocasiones digno continuador de ellos, observando una conducta ejemplar. (Enciclopedia Álvarez, 1997; citado en Castro \& Blanco, 2006, p. 238)

Este extracto ilustra algunas de las cuestiones apuntadas hasta ahora. En primer lugar, se ve cómo 
España, en tanto actor colectivo, aparece como protagonista e hilo conductor del relato, estableciendo así una continuidad narrativa denle el tiempo. También se observa una visión monocausal de la historia, basada en la supuesta identidad católica de España, identidad que articularía, a su vez, la lógica binaria de enfrentamiento con distintos enemigos del catolicismo a lo largo del tiempo: desde los árabes a Napoleón, pasando por los comunistas durante la Guerra Civil. De hecho, la propaganda franquista utilizó la idea de la "Reconquista" como temática narrativa desde la que justificar el llamado Alzamiento o Cruzada Nacional y presentar, así, su causa como la reconquista de la identidad española supuestamente perdida bajo la República (1931-1936), considerada otro paréntesis anómalo en la historia de España. Por último, al igual que en el discurso de Aznar del inicio, este fragmento proporciona también un guion orientado hacia el futuro, instando a sus lectores a seguir con el ejemplo de aquellos que en el pasado lucharon por defender la identidad española.

Tomando este ejemplo, se puede decir que el discurso del expresidente español no se produce en un vacío cultural, sino que en él se retoma y reactualiza nuevamente la temática de la Reconquista como marco narrativo para interpretar otro tipo de eventos, en este caso, ocurridos con más de 500 años de diferencia, como los atentados de Madrid de 2004. Obviamente, la enseñanza de la historia ha experimentado importantes cambios en las últimas décadas, al calor de las crecientes críticas sobre la interpretación nacionalista del pasado (Symcox \& Wilschut, 2009). España no ha sido una excepción. Tras la transición democrática con la muerte de Franco en 1975, los libros de texto han prescindido de la nostalgia imperial, volviendo a la tradición historiográfica liberal u optando por la historiografía inspirada en la Escuela Francesa de los Anales (López-Facal, 2010). En este sentido, se puede observar un gran avance; sin embargo, nociones esencialistas en torno a la identidad y al territorio nacional continúan abundando, ya sea en el modo en el que el profesorado reinterpreta y reconstruye los contenidos de los textos escolares durante la interacción en el aula (Castro \& Rasskin-Gutman, 2012; Rasskin-Gutman, 2012); en el modo en el que el alumnado sigue entendiendo el periodo de Al-Andalus en la Península Ibérica, tal como ha documentado ampliamente el equipo dirigido por Mario Carretero (López, Carretero \& Rodríguez-Moneo, 2015); o en el uso de discursos históricos y políticos pronunciados en la esfera pública (Brescó \& Rasskin-Gutman, 2006).

El trabajo que se presenta a continuación pretende examinar, a partir de un estudio de caso, hasta qué punto los libros de texto escolares se han alejado de la temática de la Reconquista o, por el contrario, siguen mediando una forma de entender la identidad (y la alteridad) española en términos esencialistas. Para ello, tal y como se apuntaba al inicio, este estudio se servirá de herramientas procedentes del análisis del discurso y del análisis visual. A este respecto, cabe aclarar que, si bien el análisis que se presenta en este artículo se centra en un nivel lingüístico-textual, no hay que olvidar la importancia del lugar de enunciación, el posicionamiento del autor y las negociaciones establecidas entre distintos participantes, así como las relaciones entre estas dimensiones. Se entiende el discurso como parte de un sistema de prácticas culturales, socio-materiales e institucionales que lo producen, reivindican, difunden y lo hacen posible (Foucault, 1969/1978; Fairclough \& Wodak, 1997; Martín Rojo, 2001). El discurso apunta a formas culturales compartidas, a modo de narrative templates, cuya autoridad se halla segregada entre aquél que lo actualiza y las instituciones y estructuras sociales que lo posibilitan. La autoría de cualquier práctica discursiva, por tanto, va más allá del locutor o emisor material del mismo, al remitir a lugares de enunciación inmersos en estructuras que restringen las prácticas discursivas posibles, las temáticas que pueden ser escogidas e, incluso, su interpretación. La estructura, pues, crea las condiciones de posibilidad para que ciertos 
discursos sean puestos en marcha, dirigiendo y regulando las acciones de los individuos, que, al mismo tiempo, disponen de agencialidad para apropiarse o, incluso, resistirse a ellos (van Alphen, en prensa).

\section{Método}

Los datos que se presentan en este trabajo forman parte de un proyecto I $+\mathrm{D}$ desarrollado durante los años 2003-2007, ${ }^{3}$ a partir de la realización de cuatro tesis doctorales. Mediante dicho proyecto, se tuvo la posibilidad de acceder a cuatro institutos de educación secundaria de la Comunidad de Madrid. El objetivo general consistió en observar qué papel cumplían las prácticas lingüísticas dentro del proceso de gestión de la diversidad - en los distintos programas de atención de esta-que ofrecía en ese momento la Comunidad de Madrid en los centros de educación secundaria de titularidad pública. De esta manera, la investigación se centró en la forma en que cada centro producía y gestionaba la diversidad lingüística, étnico-cultural y fenotípica. Teniendo foco, en especial, en la incorporación del alumnado de origen inmigrante. Para ello, desde una perspectiva cualitativa, se utilizó una estrategia etnográfica general, siguiendo la corriente de las etnografías escolares contemporáneas que se gestan fuera del campo específico de la antropología, abriéndose a otros campos sociales como la Psicología, la Sociología, la Sociolingüística y la Educación, tomando como escenario central, en este caso, la escuela (Willis 1978; Yon, 2000; Wortham, 2006; Martín Rojo \& Mijares, 2007). La etnografía escolar constituye, en sí misma, un género propio dentro de la etnografía como estrategia para el estudio contextualizado de

Proyecto de investigación Análisis sociopragmático de la comunicación intercultural en las prácticas educativas: hacia la integración en el aula, dirigido por la Dra. Luisa Martín Rojo, y financiado por los Programas de Investigación y Desarrollo (I+D) del Ministerio de Ciencia y Tecnología (MCYT) de España (Código: BFF2003 04830). fenómenos sociales (Ogbu, 2003). En esta investigación, el empleo de esta estrategia etnográfica se concretó en distintas técnicas de recogida de información, como la observación participante, las entrevistas, los grupos de discusión, el análisis de material didáctico — principalmente libros de texto- $\mathrm{y}$ análisis de documentos normativos $\mathrm{y}$ reguladores de la diversidad cultural en los centros educativos.

Por motivos de espacio, en este artículo solo se centrará en el análisis del papel mediador del libro de texto escolar ${ }^{4}$ empleado en una clase de Ciencias Sociales de 2. ${ }^{\circ}$, en un Instituto de Educación Secundaria de titularidad pública, ubicado en un pequeño municipio del sudoeste de la Comunidad de Madrid. De forma específica, se analizará el bloque histórico de dicho manual, haciendo foco, en especial, en la unidad didáctica titulada "Al-Andalus", escogida por el papel fundamental que la narrativa de este periodo histórico (711-1942) sigue cumpliendo, aún a día de hoy, en la configuración de la identidad española. Como ya se ha mencionado anteriormente, para el análisis de los contenidos del libro de texto se empleará el concepto de schematic narrative template (Wertsch, 2002), así como otros conceptos procedentes del análisis del discurso (van Dijk, 2003; Martín Rojo, 2008) y del análisis visual (van Leeuwen, 2001).

El análisis de las propuestas del libro de texto supone una fuente de información clave para comprender la complejidad de los procesos de construcción de un imaginario colectivo. Como se trabajará en el siguiente apartado, la temática de la unidad de "Al-Andalus" en el libro de texto se desarrolla entre dos polos, que irían desde lo que se ha denominado 'otro-exótico-admirable' (centrado en el conocimiento filosófico-científico

\footnotetext{
$4 \quad$ En otros trabajos previos se puede ver un análisis sobre cómo el profesorado reinterpreta y reconstruye los contenidos de este mismo texto escolar durante la interacción en el aula (Castro \& Rasskin-Gutman, 2012; Rasskin-Gutman, 2012).
} 
y el patrimonio artístico legado) y 'otro-exótico-amenazante' (centrado en la religión y algunas de sus costumbres, así como los enfrentamientos con el colectivo cristiano). La consideración de ese espacio histórico protagonizado por 'otros', así como el establecimiento de un cronotopo exótico, convierten al periodo de Al-Andalus en algo ajeno, alejado de aquello considerado como propio o parte del 'nosotros'.

\section{"Al-Andalus": un periodo marcado por la alteridad}

El libro de texto de Ciencias Sociales de $2 .^{\circ}$ de la ESO (Educación Secundaria Obligatoria en el sistema de enseñanza español), objeto de análisis,s se divide en tres bloques. Los dos primeros están dedicados al desarrollo de contenido histórico: (1) Europa en la Edad Media y (2) La Península Ibérica en la Edad Media, y el tercero, a contenido geopolítico y social: (3) Geografía del Mundo Actual. La unidad temática de "Al-Andalus", foco del análisis de esta investigación, se inserta dentro del segundo bloque, concretamente, en el tema 6. El libro dedica a este tema, en el que se explica la llegada y el asentamiento de los musulmanes en gran parte de la Península Ibérica, así como la evolución de su dominio militar y cultural durante un periodo de ocho siglos, un total de diecisiete páginas. Los tres temas restantes que componen este mismo bloque de contenido histórico se centran en la creación, evolución y, finalmente, el dominio militar de los reinos cristianos dentro del territorio peninsular. Estos tres temas, titulados "Los primeros reinos cristianos. El reino de León y el de Castilla" (tema 7), "La Corona de Castilla. Siglos XII-XV" (tema 8) y "La Corona de Aragón. Siglos XII-XV" (tema 9), abarcan aproximadamente el mismo periodo (del 722 al 1472) que la unidad de "Al-Andalus"; sin embargo, en el libro de texto, estos temas se abordan a lo largo de un total de 46 páginas.

Esta notable diferencia en el tratamiento de estos acontecimientos históricos, como expone Martínez
(2010), acentúa el papel secundario que en España se suele otorgar a Al-Andalus dentro de la configuración de la memoria y la identidad colectiva española frente a la de los mencionados reinos cristianos. La religión constituye el eje principal por medio del que se articula la propuesta identitaria que se ha observado en el libro de texto, responsable tanto de los procesos de alterización enriquecedores, que se materializan en la construcción de lo que se denomina "otro-exótico-admirable", como de los procesos de alterización antagónicos, responsables de la construcción de un "otro-exótico-amenazante". Como se verá a continuación, la distinta manera en la que son abordadas las tres grandes religiones islamismo, cristianismo y judaísmo-,${ }^{5}$ da cuenta de la propuesta identitaria de este libro de texto.

\section{La construcción del "otro-exótico- amenazante"}

Como parte del primer bloque, "Europa en la Edad Media", y dentro del primer tema "De la Antigüedad a la Edad Media”, el Islam ocupa una sección completa de dos páginas. Esta comienza con una narración descriptiva del origen de la nueva fe, es decir, dónde surge, en qué época, quiénes poblaban la región, qué religión estaba establecida en ese entonces y quién promovió el cambio religioso. Asimismo, se mencionan la Hégira y la llamada a la guerra santa como hitos históricos para el establecimiento y expansión del Islam, respectivamente. El texto continúa enumerando los cinco pilares básicos de esta religión, representada como una creencia distante y ajena a un "nosotros" que se infiere en el texto. A pesar de que en ningún momento se emplean de forma explícita recursos comparativos, la mención de aspectos

El papel que ocupa el judaísmo en el libro de texto no alcanza siquiera para configurar un "otro-exótico". Más bien, se construye el judaísmo como un "otro-ausente", o minimizado (Morales \& Lischinsky, 2008; Martín Rojo, 2010) que, por cuestiones de espacio, no se desarrollará en este artículo. 
como los que aparecen en el siguiente fragmento dan cuenta de una distancia o polarización entre un “ellos-musulmán” y un "nosotros-no musulmán”:

El Islam también establece normas de comportamiento: permite la poligamia, define la autoridad del padre dentro de la familia y prohíbe beber alcohol, comer carne de cerdo y los juegos de azar. Todo musulmán debe ser un combatiente de la fe y se le recompensa con el paraíso si muere luchando contra los infieles. (García Sebastián, 2004, p. 14) (énfasis propio)

Es importante subrayar aquí la función que cumplen, en términos identitarios, algunos elementos utilizados en dicho enunciado. La mención tanto de "la poligamia" como de "la autoridad patriarcal", como formas características o representativas de este credo religioso, en contraste con las prácticas locales que, teóricamente, prevalecen en la sociedad española actual, cumplen una función discursiva claramente diferenciadora, de deslindamiento y rechazo (van Dijk, 2003). Estas características, al ser consideradas elementos propios de sociedades en vías de desarrollo, alejadas, en principio, de la imagen que hoy en día proyecta la sociedad española, son susceptibles de provocar en el lector tanto extrañamiento como rechazo. De esta manera, se proyecta una imagen del Islam de irracionalidad y primitivismo contraria al progreso. Asimismo, la mención sobre la prohibición del consumo de alcohol y carne de cerdo (en un país, como España, en el que productos como el vino y el jamón no solo forman parte del paisaje gastronómico, sino que son, además, empleados como símbolos de la identidad colectiva nacional) se suma a la función de deslindamiento antes mencionada.

La elección léxico-metafórica relacionada con la guerra - "combatientes", "muere", "luchando" - termina por incidir, si cabe aún más, en esa función de distanciamiento, extrañamiento y rechazo ante el Islam, reforzada al mismo tiempo por el contexto histórico y local en el que este contenido está siendo recibido. En este sentido, encontramos relaciones intertextuales entre el contenido de este apartado y la información que en el momento de la publicación del libro de texto circulaba en los medios de comunicación españoles sobre el Islam. ${ }^{6}$ La mención en el texto de la llamada "lucha contra el infiel" y la "recompensa del paraíso" refuerza una visión del Islam como una religión violenta e intolerante, que la vincularía a los ataques terroristas como el referido en el discurso de Aznar del inicio. Lejos de facilitar la inclusión de diferentes perspectivas, o de generar un pensamiento complejo acerca de esta creencia religiosa, el libro de texto promueve una imagen rígida y caricaturesca, carente de matices, generadora de barreras y distanciamiento.

Asimismo, el empleo de un tiempo verbal en presente ("contiene", "define", "obliga", "establece", "permite", "define", "prohíbe", "debe ser", "se le recompensa", "está dirigida") da a entender la universalidad y vigencia actual de las normas religiosas del Islam sin modificación ni matiz alguno. A este respecto, en términos identitarios y en línea con una de las características de las narrative templates señaladas anteriormente, cabría destacar la continuidad establecida en el libro de texto entre el pasado y el presente del Islam, en tanto sujeto histórico homogéneo y permanente a lo largo del tiempo. En esta línea la fotografía que acompaña al texto refuerza también la visión de un "otro-exótico", ajeno e imperturbable al paso del tiempo. Se trata de una imagen panorámica actual de la peregrinación a la Meca en la que participan un elevado número de fieles. La representación grupal de una serie de acciones sincronizadas y la toma de "plano largo" (long shot) constituyen recursos visuales que contribuyen a reducir la individualidad $y$,

\footnotetext{
El ataque a las Torres Gemelas de Nueva York del 11 de septiembre de 2001 constituye uno de los acontecimientos clave en la proliferación de información acerca del Islam, por medio de los medios de comunicación.
} 
por ende, la emergencia de tipos (types) que refuerzan el efecto de "todos "ellos' son lo mismo" (van Leeuwen, 2001, p. 96). El empleo de estos recursos forma parte, en términos de van Leeuwen, de una estrategia discursiva que favorece la generalización, tipificación y estereotipación de dicho colectivo.

\section{La construcción del "nosotros"}

Desde el comienzo de los bloques de contenido histórico, el cristianismo aparece como un elemento central en la mayor parte de las unidades. Se dice de los pueblos germánicos — visigodos, francos, vándalos, ostrogodos, anglos y sajones - que, "tenían una cultura menos desarrollada que la romana [y que], pronto fueron dominados por el espíritu romano: adoptaron el cristianismo como religión y el latín como lengua" (García, 2004, p. 6). Asimismo, se dedica una sección específica a Bizancio al que se describe como un imperio cristiano, destacando el papel central de la Iglesia. Se estudian los legados artísticos y culturales de la Edad Media, estrechamente vinculados a la religión cristiana y, de nuevo, su Iglesia. Además, como ya se indicó anteriormente, se dedican tres temas específicos a la explicación de la creación y evolución de los reinos cristianos en la Península Ibérica.

Esta omnipresencia de "lo cristiano", a diferencia de lo que ocurría con "lo musulmán", indica el papel fundamental que se le otorga a esta religión en la construcción de la identidad, no solo nacional sino, al mismo tiempo, europea. De esta manera, vuelve a quedar en evidencia el carácter anecdótico del periodo histórico de Al-Andalus que, como ya se dijo, queda reducido a una sola unidad con el añadido de una sección específica sobre el Islam y otra sobre el Califato como imperio islámico. La información sobre la religión cristiana equivalente a la que ya se ha analizado sobre el Islam, aparece en el libro de texto en relación con el papel de la Iglesia durante la Edad Media. En el primer bloque, dentro del tema 3: "La Cristiandad. Cultura y arte del Románico", se encontró un pequeño apartado de dos párrafos titulado "Una Europa Cristiana" en la que se define el papel de la Iglesia, así como los preceptos religiosos que esta prescribe en términos pretéritos:

La Iglesia se encargaba de fijar las obligaciones religiosas de los cristianos y de mantener su unidad. Debían rezar, asistir a la misa del domingo, ayunar en días señalados, confesarse una vez al año, comulgar por Pascua y realizar, si era posible, peregrinaje a un lugar santo (Roma, Jerusalén, Santiago de Compostela [...]) en el que se conservan reliquias de Cristo o de los mártires y santos cristianos. Igualmente se prescribían la caridad, la vida virtuosa y la donación de bienes a la Iglesia en el momento de la muerte. [...] Todo ello servía para redimir las faltas o pecados y alcanzar la vida eterna. (García, 2004, p. 42; énfasis nuestro)

En el apartado "Para Recordar", se continúa utilizando este mismo recurso discursivo: "La Iglesia tuvo una enorme influencia y poder en la Edad Media: intervenía en la vida social y regulaba la vida espiritual de los creyentes, imponiéndoles obligaciones religiosas" (García, 2004,p. 55; énfasis nuestro). La elección del tiempo verbal pretérito en el caso del papel de la Iglesia y del tiempo presente en el caso del Islam no es casual. Refuerza, por un lado, la idea de inmovilismo del Islam respecto al Cristianismo sobre la separación religión-estado y, por otro, la promoción de una mirada esencialista mediante la que parece posible saber "lo que realmente se dice en el Corán", sin considerar la mirada e intereses interpretativos existentes en cada lugar y momento histórico Maalouf (2009).

\section{La Construcción del "Otro-Exótico- Admirable"}

Junto con la consideración del "otro-amenazante", la alteridad que subyace a la descripción de Al-Andalus también aparece, en clave positiva, 
como el "otro exótico y admirable". Para empezar, se interpreta su llegada y asentamiento en la Península como una acción cultural y civilizadora, en la que se supera el carácter rural de los reinos cristianos para pasar a un carácter más urbano. Asimismo, se ofrece una imagen de este colectivo como transmisor de nuevos conocimientos y enlace con otras culturas. Se destaca su función de "puente de unión entre la herencia de la Antigüedad clásica y la Europa medieval" (García, 2004, p. 98), mencionando la recuperación y traducción de obras filosóficas y científicas de la Antigua Grecia, así como la importación desde Oriente de técnicas de producción tales como el papel. En cuanto a producciones propias, se menciona la numeración arábiga y se señala, aunque sin dar ninguna especificación concreta, el desarrollo que produjeron en disciplinas como la Medicina, la Astronomía o la Botánica, entre muchas otras. En la figura de Averroes, filósofo musulmán del siglo XII, quedan condensados todos estos valores de producción y transmisión de conocimiento. Mediante todos estos datos, el libro confiere a "la cultura musulmana" una imagen de superioridad frente a unos "pueblos europeos", de los que no se especifica nada concreto, restando énfasis a los aspectos que, por contraste, pudieran ser negativos para la imagen de estos últimos (Van Dijk, 2003). De esta manera, a un nivel específicamente cultural, en el libro de texto no solo se valora de manera positiva la presencia de este colectivo en la Península sino que, además, este es admirado:

Por esta razón [sus conocimientos acerca de las obras científicas y filosóficas producidas en la Antigua Grecia] la superioridad cultural de los árabes era evidente y durante los siglos que dominaron la Península transmitieron a los pueblos europeos el conocimiento de la ciencia griega por medio de traducciones y comentarios. (García, 2004, p. 98)

El apartado relativo al arte árabe queda limitado a la descripción de su arquitectura, con abundantes referencias y fotografías a lo largo de toda la unidad. La prohibición de las representaciones humanas, presente en el Corán, se utiliza en el texto como argumento para justificar la ausencia de información relativa a otras formas de expresión artística: "Por este motivo cuando hablamos de arte árabe nos referimos casi exclusivamente a la arquitectura" (García, 2004, p. 99). Respecto a la economía, se destacan las mejoras en la agricultura incorporadas por los nuevos pobladores, a los que en este caso el libro de texto hace referencia mediante el apelativo "andalusíes", como, por ejemplo, la noria (técnica de regadío) o la introducción de nuevos cultivos, como el arroz o la naranja, entre otros. Se subraya también la importancia de Al-Andalus como centro de intercambio comercial entre Europa y Oriente. Con todo ello, el balance que en el libro se hace de la llegada de los musulmanes a la Península es completamente favorable; no obstante, como se viene diciendo, este se construye como un colectivo aparte que no se integra dentro del imaginario de un "nosotros" pretérito. Respecto a la incorporación en el texto y empleo de categorías como "andalusí", en apariencia inclusiva, "conquistadores" e "hispanos", se hablará brevemente en el siguiente apartado.

\section{'Conquistadores', 'hispanos' y 'andalusíes'}

A lo largo de esta la unidad de "Al-Andalus", se emplean diversas etiquetas como forma de caracterizar a diferentes agentes colectivos. Estos agentes aparecen como protagonistas de los acontecimientos históricos narrados y, por tanto, es interesante analizar qué papel cumplen en la configuración de la propuesta identitaria del libro. En este sentido, Al-Andalus se representa como una sociedad diversa y mestiza: "La sociedad de Al-Andalus estaba formada por una mezcla de gentes de distintas razas, culturas y religiones. Los mismos conquistadores eran diferentes entre sí, aunque todos eran musulmanes de religión" 
(García, 2004, p. 96). De esa mezcla de gentes, en el libro se establecen diferencias entre 'conquistadores' e 'hispanos'.

Bajo la etiqueta de 'conquistadores', se hallan agrupados los árabes, los sirios y los bereberes. Este grupo, unido por un criterio religioso, muestra su heterogeneidad a partir del establecimiento de un criterio de clase social. De esta manera, en la unidad de "Al-Andalus", se dice que los árabes y los sirios pertenecían a una élite de poder, mientras que los bereberes ocupaban un escalafón más bajo. Por su parte, la categoría 'hispanos' une a grupos diversos a partir de un criterio territorial y, por lo tanto, es de suyo heterogénea. Esta categoría contempla a muladíes, mozárabes y judíos, diferenciados entre ellos, a su vez, a partir de un criterio religioso, ya sea por continuidad, como en el caso de los judíos y los mozárabes, o por conversión, como en el caso de los muladíes.

Por último, la etiqueta "andalusí", mencionada tan solo en tres ocasiones a propósito del comercio y del legado de los intelectuales de la época, se infiere como una categoría que engloba tanto a conquistadores como a hispanos. No obstante, la ausencia de definición explícita del término, así como el uso ambivalente del mismo, puede dar a entender que este se refiere exclusivamente a los nuevos pobladores musulmanes y, por tanto, no a la sociedad de aquella época en su conjunto:

Entre los siglos VIII y XV la base principal de la economía de Al-Andalus fue la agricultura. Los andalusíes introdujeron novedades importantes:

[...] nuevas técnicas de regadío, como la noria. [...] nuevos cultivos como el arroz, la naranja, la morera, el cáñamo y el azafrán [...]. (García, 2004, 96; énfasis nuestro)

Lo que resulta común al empleo de estas tres categorías es que, en todos los casos, se ofrece una definición concisa y, por ello, carente de complejidad, en la que, además, se omite información de tipo relacional. Es decir, se omiten descripciones de costumbres y formas de relación tanto dentro de cada grupo como con otros grupos, por lo que el lector no obtiene imagen alguna acerca del tipo de intercambios existentes entre estos agentes y, tampoco, de cuál fue su evolución en el tiempo. Respecto a esta ausencia de elementos relacionales, resultan de interés las observaciones de Michael Apple (1986), quien señala que la mayoría de los currículos escolares se diseñan alrededor de la idea de consenso; algo que "guarda poca semejanza con la complejidad del nexo y las contradicciones que rodean al control y organización de la vida social" (pp. 18-19). La manera de tratar la información en esta unidad no es diferente a lo que señala Apple. Como se dijo, en ella se omite información relativa a los conflictos entre los distintos grupos, excepto en el caso de los mozárabes (cristianos que habitaban en territorio musulmán conservando su religión), en cuyo caso, se alude a persecuciones religiosas que, según se menciona en el libro de texto les empujó a emigrar hacia territorio cristiano.

Los únicos datos de tipo relacional aparecen en la siguiente unidad: "Los primeros reinos cristianos. El reino de León y el de Castilla", en un apartado titulado "La Península Ibérica: encuentro de culturas". A pesar de que se alude a la existencia de periodos de enfrentamiento entre cristianos, musulmanes y judíos, el texto solo destaca los ejemplos de convivencia, mitigando, de esta manera, el peso de lo conflictivo. En general, el libro refuerza una imagen de coexistencia armónica, ensalzando, por un lado, un supuesto pasado "ejemplar", condensado en las famosas Escuelas de Traducción de Toledo, y eludiendo, por otro, las complejidades de la relación social entre los distintos grupos y su evolución en el tiempo.

\section{El término "reconquista" y sus implicaciones identitarias}

Para finalizar, es clave analizar el uso que en este se hace del término 'Reconquista'. Esta etiqueta histórica, como ya se ha indicado, ha sido 
utilizada como forma de ensalzar uno de los hitos fundamentales del relato de génesis de la nación española, promoviendo la idea de que Al-Andalus solo supuso un "factor presuntamente anómalo y alterador de una visión lineal, unidimensional, esencialista [de la identidad española]" (Martínez, 2010, p. 21). De este modo, el concepto 'Reconquista', actualmente en desuso en la mayoría de manuales escolares y en debate entre los historiadores medievales (Barbero \& Vigil, 1974; Ríos Saloma, 2013;), proyecta una imagen cohesionada de los diferentes grupos de pobladores que, desde el norte de la Península, lucharon contra los musulmanes. A estos pobladores, además, y siguiendo una lógica interesada de continuidad histórica típica de las narrative templates, tradicionalmente se les ha considerado como antepasados de los españoles en la actualidad.

El empleo moderado que se hace de esta etiqueta histórica en el libro de texto parece hacerse eco de este debate, hasta el punto de evitarlo durante la unidad de "Al-Andalus", donde el avance de los reinos cristianos es referido en el texto como "conquista" de territorios, nunca como 'Reconquista'. Asimismo, en esta unidad, se habla de la conquista musulmana en términos de 'llegada' y no de invasión, lo cual mitiga la imagen amenazante de este periodo para la identidad colectiva: "La llegada del ejército, al mando de Tariq, había sido provocada por los mismos visigodos, pues habían pedido ayuda a los musulmanes del Norte de África contra el rey don Rodrigo" (García, 2004, p. 90; énfasis nuestro).

Sin embargo, en las siguientes tres unidades que completan el segundo bloque, titulado: "La Península Ibérica en la Edad Media", centradas en la creación, consolidación y avance de los reinos cristianos, los términos empleados cambian por completo. Así, la llegada de los musulmanes referida en la unidad anterior se convertirá en invasión: "Los ejércitos islámicos que habían invadido la Península Ibérica en el año 711 no alcanzaron a dominar plenamente todo su territorio" (García,
2004, p. 108; énfasis nuestro). Asimismo, la 'conquista' cristiana de la Península, será referida, esta vez, en términos de 'Reconquista': "[La batalla de Covadonga] ha sido considerada tradicionalmente como el inicio de la Reconquista, es decir, de la conquista por los reinos cristianos de las tierras peninsulares ocupadas por los musulmanes" (García, 2004, p. 108; énfasis del original).

Si bien este término no aparece como encabezamiento de ningún apartado, de forma que no protagoniza el discurso histórico, cabe destacar que en el libro se hace referencia al término 'Reconquista' en múltiples ocasiones y, de forma recurrente, en los apartados "Para Recordar" que aparecen al final de las tres unidades relativas a los reinos cristianos, como puede verse en el siguiente ejemplo: "La Reconquista y la repoblación realizada por Castilla avanzó mucho a lo largo de los siglos XII y XIII. A finales del siglo XIII ya solo quedaba en la Península Ibérica un reino musulmán, el reino nazarí de Granada" (García, 2004, sección "Para Recordar", p. 139). El diferente empleo de este término a lo largo del segundo bloque de contenido histórico podría indicar, simplemente, la distinta autoría de cada capítulo. No obstante, la falta de consenso en la utilización o no de este término ya es un indicador de una toma de conciencia, aunque sea incipiente, de las connotaciones o implicaciones identitarias que subyacen a esta etiqueta histórica. El hecho de no aparecer como encabezamiento de ningún apartado ya supone un avance con respecto al uso tradicional que, hasta hace poco, venía haciéndose en libros de texto españoles.

\section{Discusión}

Los resultados del estudio muestran cómo el libro de texto analizado, aun dejando claramente atrás las propuestas esencialistas de la Enciclopedia Álvarez o el discurso de Aznar del inicio, continúa reproduciendo, en determinados puntos, una visión de Al-Andalus no exenta de cierta carga identitaria. El concepto de 'Reconquista' sigue 
apareciendo, en ocasiones, como un hito en el relato, proyectando, sobre la base de una identidad cristiana, una cierta continuidad histórica entre los grupos de pobladores que lucharon contra los musulmanes y los españoles actuales. Se ve, pues, cómo mediante esta forma de tramar el pasado se vehicula implícitamente la noción de un sujeto histórico homogéneo y permanente en el tiempo. Si bien durante la unidad de "Al-Andalus" se evita el empleo de este concepto, vemos cómo el término "Reconquista" sí aparece en las unidades sobre la creación de los Reinos Cristianos, incidiendo así en la tendencia a conceptualizar la identidad y el territorio en términos esencialistas, en línea con resultados obtenidos en otras investigaciones con estudiantes y su concepto de nación (López, Carretero \& Rodríguez-Moneo, 2015).

Por su parte, el pasado musulmán de la Península Ibérica sigue representándose, en cierta medida, en clave de alteridad, ya sea en términos amenazantes o elogiando las aportaciones y el desarrollo procedentes de una cultura representada como exótica y ajena. Nuevamente, aparece, aunque de forma atenuada, otra de las características típicas de las narrative templates mencionadas al inicio, a saber, una lógica binaria, articulada mediante la presencia de un 'nosotros' y un 'ellos', sin apenas información relacional, omitiendo formas de relación e intercambio entre los grupos. Si bien tal planteamiento ha ido cambiando notablemente durante los últimos lustros, con la progresiva incorporación de alumnado de otros países en las aulas españolas, esta forma de organizar discursivamente el texto naturaliza procesos de alterización que en nada contribuyen a la configuración y comprensión de sociedades interculturales (Rasskin-Gutman, 2015).

En resumen, se evidencia cómo los procesos de producción de identidad (y alteridad) articulados en la vieja temática de la 'Reconquista' se anclan en formas de tramar el pasado fuertemente arraigadas en el modo de entender la identidad colectiva. Desde la perspectiva de la psicología cultural, se podría decir que tales formas de imaginar el pasado y la identidad vendrían mediadas por aquellas herramientas simbólicas disponibles en una determinada comunidad. En este caso de análisis, la idea de 'Reconquista' —incluyendo las nociones de identidad y alteridad que la propia idea vehicula - continúa incorporada a todo un sistema de prácticas culturales e institucionales en España, desde monumentos, celebraciones y desfiles populares, pasando por libros de texto y mapas escolares. Se trata, en definitiva, de prácticas que, con niveles distintos de reflexión y crítica, continúan reproduciendo, en menor o mayor medida, una determinada forma de narrar el pasado histórico. En este sentido, siguiendo los planteamientos de Bakhtin (1981), se entiende que la voz — ya sea la de los libros de texto o la de los discursos políticos - no tiene una sola autoría, sino que esta es compartida por las distintas formas narrativas heredadas en un determinado grupo, las cuales se erigirían, en cierto modo, como co-autoras de los discursos que se construyen y circulan en él.

\section{Conclusiones}

En septiembre de 2006, dos años después del discurso en la Universidad de Georgetown, José María Aznar afirmaba, en el Hudson Institute de Washington, no haber "oído a ningún musulmán pedir disculpas por haber conquistado España y por haber mantenido su presencia durante ocho siglos". ${ }^{7}$ En ambos casos, se evidencia cómo episodios lejanos del pasado siguen vivos en el presente, cómo la llamada 'Reconquista', no solo continúa siendo un hito histórico para la concepción de la identidad española, sino que opera, además, como tema narrativo para interpretar el presente y el futuro, especialmente en tiempos de incertidumbre. En este sentido, no es casual que el lema electoral del emergente partido de

Discurso pronunciado en la conferencia Global Threats, Atlantic Structures, celebrada en el Hudson Institute, Washington DC, el 21 de septiembre de 2006. 
ultraderecha en las elecciones generales de 2019 haya sido el de la 'Reconquista de España', y que su inicio de campaña haya sido en Covadonga (Principado de Asturias) en conmemoración de Don Pelayo, caudillo astur al que se le atribuye el inicio de la Reconquista, en el año 722, en la batalla de Covadonga. Nuevamente, la vieja temática de la Reconquista es utilizada — en este caso, contra progresistas, catalanistas, islamistas y comunistas - como herramienta simbólica para la movilización en tiempos de crisis; como un guion fácil de entender debido, precisamente, a su fuerte implantación en la memoria colectiva del país.

Como se ha señalado, las narrative templates, en tanto discursos dominantes, proyectan escenarios en los que se asignan distintos roles basados en supuestas esencias identitarias, cuya interiorización puede acabar convirtiéndonos en actores sujetos a guiones culturalmente heredados (véase Liu \& Hilton, 2005). Si bien los libros de texto tienden a plantear una visión de la historia cada vez más crítica y reflexiva, y, por tanto, más acorde con unas aulas cada vez más multiculturales, se ha mostrado la pervivencia de determinadas temáticas narrativas fuertemente arraigadas en la sociedad. A este respecto, se entiende que la enseñanza de la historia, lejos de alentar la transmisión de identidades cerradas y excluyentes, debería transmitir las herramientas necesarias para que los individuos dejen de ser actores y se conviertan en autores con capacidad crítica para construir y negociar versiones alternativas del pasado y, con ello, poder imaginar otras formas de identidad posibles. En esta línea, diversos autores (Barton \& Levstik, 2004; Egan, 1997/2000; Rosa \& Brescó, 2018) apuestan por hacer visibles las narrativas que median los distintos modos de reconstruir el pasado para tomar conciencia de las temáticas que transmiten, de los escenarios que proyectan $\mathrm{y}$, especialmente, de las identidades y alteridades que naturalizan. En este sentido, se considera que las herramientas analíticas que provienen del análisis del discurso, especialmente de las corrientes críticas (Fairclough \& Wodak, 1997; Martín Rojo, 2001), pueden ser de gran ayuda para este cometido, tal y como se espera haber mostrado con los análisis presentados en este trabajo.

\section{Referencias}

Álvarez, J. J. (2002). The formation of Spanish identity and its adaptation to the age of nations. History \& Memory, 14(1/2), 13-36.

Anderson, B. (1983). Imagined communities. Londres: Verso.

Apple, M. W. (1986). Ideología y currículo. Madrid: Akal.

Aznar, J. M. (2004, septiembre, 21). Seven theses on today's terrorism. Discurso pronunciado en Georgetown University, Washington, DC.

Bakhtin, M. M. (1981). The dialogic imagination: Four essays by M. M. Bakhtin (C. Emerson \& M. Holquist, Trad.) Austin, TX: University of Texas Press.

Barbero, E., \& Vigil, M. (1974). Sobre los orígenes sociales de la reconquista. Barcelona: Madrid.

Barth, F. (1969/1976). Los grupos étnicos y sus fronteras. La organización social de las diferencias culturales. México: Fondo de Cultura Económica.

Barton, K., \& Levstik, L. S. (2004). Teaching history for the common good. Mahwah. New Jersey, N.J.: LEA.

Billig, M. (1995). Banal nationalism. Londres: Sage.

Boyd, C. P. (2003). El debate sobre "la nación" en los libros de texto. En J. J. Carreras \& C. Forcadell (Eds.), Usos de la historia (pp. 145-171). Madrid: Marcial Pons.

Brescó, I. (2008). Giving national form to the content of the past. A study of the narrative construction of historical events. Journal of Psychology and Society, 1(1), 1-14.

Brescó, I., \& Rasskin-Gutman, I. (2006). Función y usos (¿abusos?) de los discursos históricos en la construcción social de la identidad. Revista de historia de la Psicología, 27, 371-379. 
Brescó, I., \& Wagoner, B. (2019). The psychology of modern memorials: The affective intertwining of personal and collective memories / $\mathrm{La}$ psicología de los monumentos modernos: la implicación afectiva de los recuerdos personales y colectivos. Estudios de Psicología, 40(1), 219-244. Doi: 10.1080/02109395.20 18.1560024

Bruner, J. (1986). Actual minds and possible worlds. Cambridge, Mass: Harvard University Press.

Carretero, M. (2007). Documentos de identidad. Buenos Aires: Paidós.

Carretero, M., \& Bermúdez, A. (2012). Constructing histories. En J. Valsiner (Ed.), Oxford Handbook of Culture and Psychology (pp. 625-646). Oxford, R.U.: Oxford University Press.

Castro, J., \& Blanco, F. (2006). La trama regeneracionista: sobre el valor civilizatorio de la historia y otros cuentos. En M. Carretero, A. Rosa \& M ${ }^{\mathrm{a}}$ F. González (Comp.), Enseñanza de la historia y memoria colectiva (pp. 215-241). Buenos Aires: Paidós.

Castro, J., \& Rasskin-Gutman, I. (2012). A (re)estabilização identitária do professor no contexto do ensino de história na Espanha. Cadernos Cedes, 32(88), 285-301.

Chevallard, Y. (1991). La transposition didactique. Du savoir savant ou savoir enseigné. París: La pensée sauvage.

Cole, M. (1996/1999). Psicología cultural. Madrid: Morata.

Egan, K. (1997/2000). Mentes educadas. Cultura, instrumentos cognitivos y formas de comprensión. Barcelona: Paidós.

Fairclough, N., \& Wodak, R. (1997). Critical discourse analysis. En T. A. Van Dijk (Ed.), Discourse studies: A multidisciplinary introduction, 2, (pp. 258-284).

Foucault, M. (1969/1978). Arqueología del saber. México: Siglo XXI.
García, S. M. (2004). Limes 2 Ciencias Sociales, Geografía e Historia: Educación Secundaria Obligatoria, Segundo Curso, [1 er. Ciclo]. Barcelona: Vicens Vives.

Halbwachs, M. (1950/1980). The collective memory. Nueva York, N.Y.: Harper and Row.

Hall, S., \& Du Gay, P. (Eds.) (1996). Questions of cultural identity. Londres: Sage.

Huntington, S. P. (1996). The clash of civilizations and the remaking of world order. Nueva York, N.Y.: Simon \& Schuster.

Liu, J. H., \& Hilton, D. (2005). How the past weighs on the present: Social representations of history and their role in identity politics. British Journal of Social Psychology, 44, 537-556.

López Facal, R. (2010). Nacionalismos y europeísmos en los libros de texto: Identificación e identidad nacional. Clio \& Asociados, 14, 9-33.

López, C., Carretero, M., \& Rodríguez-Moneo, M. (2015). Conquest or reconquest? Students' conceptions of nation embedded in a historical narrative. Journal of the Learning Sciences, 24(2), 252-285. Doi: 10.1080/10508406.2014.91986

Maalouf, A. (2009). Identidades asesinas. Madrid: Alianza.

Martín Rojo, L. (2001). New developments in discourse analysis: Discourse as social practice. Folia Linguistica, ХХХV(1-2), 41-78.

Martín Rojo, L. (2008). Imposing and resisting ethnic categorization in multicultural classroms. En R. Dolón \& J. Todolí (Eds.), Analysing identities in discourse. Amsterdam: John Benjamins Publising Co.

Martín Rojo, L. (2010). Constructing inequality in multilingual classrooms. Berlín/Nueva York: De Gruyter Mouton.

Martín Rojo, L., \& Mijares, L. (Ed.) (2007). Voces del aula. Etnografias de la escuela multilingüe. Madrid: Centro de Investigación y Documentación Educativa (CIDE). 
Martínez, P. (2010). Significado y símbolo de Al-Andalus. Cantabria: Fundación Ibn Tufayl de Estudios Árabes.

Morales, O. A., \& Lischinsky, A. (2008). Discriminación a través de las ilustraciones de libros de texto de Enseñanza Secundaria Obligatoria en España. Discurso y Sociedad, 2(1), 115-152.

Moreno, A. (15 de enero de 2019). ¿Por qué Vox rescata ahora el viejo concepto de 'reconquista'? Diario Público. Recuperado de https://www. publico.es/politica/ultraderecha-vox-rescata-viejo-concepto-reconquista.html

Mosse, G. L. (1975). The nationalization of the masses. Ithaca, N.Y.: Cornell University Press.

Ogbu, J. (2003). Etnografía escolar. Una aproximación a nivel múltiple. En H. M. Velasco, F. J. García Castaño \& Á. Díaz de Rada (Eds.), Lecturas de antropología para educadores. El ámbito de la antropología de la educación y de la etnografia escolar (pp. 145-174). Madrid: Trotta.

Quiroga, A. (2007). Making Spaniards. Primo de Rivera and the nationalization of the masses, 1923-30. Nueva York: Palgrave MacMillian.

Rasskin-Gutman, I. (2012). Identidad y alteridad en la escuela multicultural: una etnografía crítica. (Tesis doctoral inédita, Universidad Autónoma de Madrid, Madrid).

Rasskin-Gutman, I. (2015). La interculturalidad como proyecto ético-político e identitario. Diagonal. Recuperado de https://www.diagonalperiodico. net/blogs/fuera-clase/la-interculturalidad-como-proyecto-etico-politico-e-identitario.html

Ricoeur, P. (1991). Narrative identity. En D. Wood (Ed.), On Paul Ricoeur: Narrative and interpretation (pp. 20-30). Londres: Routledge.

Ríos Saloma, M. F. (2013). La reconquista en la historiografía española contemporánea. Madrid: Sílex.

Rosa, A., \& Brescó, I. (2017). What history to teach when the social pact shakes? En M. Carretero,
S. Berger \& M. Grever (Eds.), Palgrave Handbook of Research in Historical Cultural and Education (pp. 413-425). Londres: Palgrave Macmillan.

Rosa, A., \& Valsiner, J. (Eds.) (2018) Cambridge Handbook of Sociocultural Psychology, $2^{\mathrm{a}} \mathrm{ed}$. (pp. 443-457). Cambridge, R.U.: Cambridge University Press.

Symcox, L., \& Wilschut, A. (Eds.) (2009). National history standards. The problem of the canon and the future of teaching of history. Charlotte, NC: Information Age Publishing.

Van Alphen, F., \& Carretero, M. (2015). The construction of the relation between national past and present in the appropriation of historical master narratives. Integrative Psychological \& Behavioral Science, 49, 512-530. Doi: 10.1007/ s12124-015-9302-x

Van Alphen, F. (en prensa). Beyond the master narrative: Memories in the globalizing educational context. En B. Wagoner, I. Brescó, \& Zadeh, S. (Eds.), Memory in the wild. Charlotte, N.C.: Info Age Publishers.

Van Dijk, T. A. (2003). Ideología y discurso. Una introducción multidisciplinaria. Barcelona: Ariel.

Van Leeuwen, T. (2001). Semiotics and iconography. En T. van Leeuwen \& C. Jewitt (Eds.), Handbook of Visual Analysis (pp. 92-118). Londres: Sage.

Vygotsky, L. S. (1978). Mind in society: The development of higher psychological processes. Cambridge, MA: Harvard University Press.

Wagner, W., Kello, K., \& Saki, I. (2018). Politics, identity, and perspectives in history textbooks. En J. Pires \& K. Van Niuwenhuyse (Eds.), Representations of colonial pasts in (post)colonial presents: Historical and social psychological perspectives through textbook analysis (pp. 31-50). Charlotte, NC: Information Age Publishers.

Wertsch, J. V. (2002). Voices of Collective Remembering. Cambridge: Cambridge University Press. 
Wertsch, J. V., \& O’Connor, K. (1994). Multivoicedness in historical representation: American college students' accounts of the origins of the U.S. Journal of Narrative and Life History, 4(4), 295-310. Doi:10.1075/jnlh.4.4.04mul

Willis, P. (1978). Aprendiendo a trabajar. cómo los chicos de la clase obrera consiguen trabajos de clase obrera. Madrid: Akal/Universitaria.
Wortham, S. (2006). Learning identity. The joint emergence of social identification and academic learning. Nueva York: Cambridge University Press.

Yon, D. A. (2000). Elusive culture schooling, race, and identity in global times. Albany, N.Y.: State University of New York Press.

\section{Recibido: junio 4, 2019 \\ Aprobado: agosto 5, 2019}

